\title{
Unusually weak electron-phonon coupling in the Shockley surface state on Pd(111)
}

\author{
I. Yu. Sklyadneva, ${ }^{1,2,3}$ R. Heid, ${ }^{3}$ V. M. Silkin, ${ }^{1}$ A. Melzer,${ }^{4}$ K. P. Bohnen, ${ }^{3}$ P. M. Echenique, ${ }^{1,5}$ Th. Fauster, ${ }^{4}$ and \\ E. V. Chulkov ${ }^{1,5}$ \\ ${ }^{1}$ Donostia International Physics Center (DIPC), Paseo de Manuel Lardizabal, 4, 20018 San Sebastián/Donostia, Basque Country, Spain \\ ${ }^{2}$ Institute of Strength Physics and Materials Science, pr. Academicheski 2/1, 634021 Tomsk, Russia \\ ${ }^{3}$ Forschungszentrum Karlsruhe, Institut für Festkörperphysik, P.O. Box 3640, D-76021 Karlsruhe, Germany \\ ${ }^{4}$ Lehrstuhl für Festkörperphysik, Universität Erlangen-Nürnberg, D-91058 Erlangen, Germany \\ ${ }^{5}$ Departamento de Física de Materiales and Centro Mixto CSIC-UPV/EHU, Facultad de Ciencias Químicas, UPV/EHU, Apartado 1072, \\ 20080 San Sebastián/Donostia, Basque Country, Spain
}

(Received 14 July 2009; published 31 July 2009)

\begin{abstract}
Electron-phonon $(e-p h)$ coupling in the $\bar{\Gamma}$ unoccupied surface state on $\operatorname{Pd}(111)$ is studied using densityfunctional perturbation-theory calculation and two-photon photoemission measurements. Also, an ab initio GW calculation is performed to combine it with the $e$-ph coupling evaluation in the study of the surface-state linewidth. We show that the $e$-ph coupling in the surface state is unusually weak. It is smaller by a factor of 5 than that at the Fermi surface of bulk Pd. Temperature-dependent two-photon photoemission measurements confirm this result indicating an important role of interband scattering from surface to bulk states.
\end{abstract}

DOI: 10.1103/PhysRevB.80.045429

PACS number(s): 73.20.- r, 63.20.kd

\section{INTRODUCTION}

Dynamics of excited electrons on metal surfaces are of paramount importance for molecular motion induced by femtosecond laser pulses, ${ }^{1}$ for energy transfer in photochemical reactions, ${ }^{2}$ for catalytical reactions, ${ }^{3}$ and for charge and spin transfer across surfaces and interfaces. ${ }^{4}$ Excited electrons interact with other electrons $(e-e)$, phonons $(e-p h)$, and defects $(e-d) .{ }^{5}$ The associated scattering processes contribute to the total linewidth $\Gamma_{t o t}=\Gamma_{e-e}+\Gamma_{e-p h}+\Gamma_{e-d}$ observed in spectroscopy. In time-resolved spectroscopy the decay of the population in a particular state can be followed directly and is described by the lifetime $\tau$. The lifetime broadening $\hbar / \tau$ is included in the total linewidth and describes the scattering of the excited electron into other states associated with a change in energy or/and momentum. Electron-electron scattering is predominantly inelastic and $\Gamma_{e-e}$ is contained in $\hbar / \tau .^{5} \operatorname{In} e$-ph and $e-d$ scattering the energy exchange is small compared to the experimental resolution. Therefore most scattering events leave the population of the observed state apparently unchanged. Contributions from $\Gamma_{e-p h}$ and $\Gamma_{e-d}$ to $\hbar / \tau$ are limited to scattering events with large momentum transfer, e. g., from surface to bulk bands. ${ }^{6}$

An estimate of $\Gamma_{e-p h}$ is normally performed by using a Debye model with the experimental $e-p h$ coupling parameter $\lambda$ measured at $E_{\mathrm{F}}$ of bulk metal. For surfaces studied up to now including noble-metal surfaces, ${ }^{5,7-10} \mathrm{Mo}(110),{ }^{11,12}$ as well as simple and semimetal surfaces ${ }^{13,14}$ this picture works quite well. Probably the only exception is beryllium whose surfaces show density of states (DOS) at $E_{\mathrm{F}}$ much higher than that in bulk Be due to the appearance of strong surface electronic states at $E_{\mathrm{F}}{ }^{15}$ In this work we show an example when the $e$-ph coupling in a Shockley surface state is very distinct from that at $E_{\mathrm{F}}$ of bulk metal despite that the bulk DOS at $E_{\mathrm{F}}$ is not very different from the surface DOS. This distinction being important at low temperature essentially affects the linewidth at elevated temperatures when $\Gamma_{e-p h}$ can significantly exceed $\hbar / \tau$. By using time-resolved two-photon photoemission Schäfer et al. ${ }^{16}$ measured the lifetime broadening of the $\bar{\Gamma}$ Shockley surface state on $\operatorname{Pd}(111), \hbar / \tau$ $=54 \mathrm{meV}$ at $T=450 \mathrm{~K}$ and the total linewidth $\Gamma_{\text {tot }}$ $=100 \mathrm{meV}$. The difference between $\Gamma_{e-e}$ and $\Gamma_{t o t}$ was attributed to the quasielastic scattering due to phonons. The theoretical estimation of the $e$ - $e$ inelastic scattering with a model that combines one-dimensional potential and many-body effects ${ }^{16}$ gave $\Gamma_{e-e}=37 \mathrm{meV}$ which is slightly smaller than the experimental values for $\hbar / \tau$. The Debye model with $e$-ph coupling parameter $\lambda=0.40$ taken from $a b$ initio calculations ${ }^{17}$ of bulk Pd gives $\Gamma_{e-p h}=60 \mathrm{meV}$ at $T=450 \mathrm{~K}$. This leads to theoretical $\hbar / \tau+\Gamma_{e-p h}=97 \mathrm{meV}$ in good agreement with the measured linewidth $\Gamma_{t o t}$ but not with the measured $\hbar / \tau$.

Here we show by performing ab initio calculations that in the unoccupied $\bar{\Gamma}$ surface state on $\operatorname{Pd}(111)$ the $e$ - $p h$ coupling parameter is very small, $\lambda=0.08$, i. e., by a factor of 5 smaller than $\lambda$ obtained at the Fermi energy of bulk Pd. ${ }^{17}$ This reduces the calculated $\Gamma_{e-p h}$ to $19 \mathrm{meV}$ and cannot explain the large difference between the experimental values for $\hbar / \tau$ and $\Gamma_{\text {tot }}$ at $T=450 \mathrm{~K}$. In order to clarify this discrepancy we performed temperature-dependent linewidth measurements by time-resolved two-photon photoemission. We also carried out an $a b$ initio evaluation of the $e$-e contribution to the lifetime broadening of the $\bar{\Gamma}$ surface state.

\section{DETAILS OF THE CALCULATION}

The e-ph calculations were performed using densityfunctional theory and the local-density approximation for the exchange-correlation functional. Phonon frequencies and polarization vectors were obtained within the mixed-basis density-functional perturbation theory ${ }^{18,19}$ using a normconserving scalar-relativistic pseudopotential. ${ }^{20}$ The planewave energy cutoff was 20 Ry and, additionally, one $d$-type localized wave function at each atomic site of Pd was employed. Integrations over the surface Brillouin zone (SBZ) 
were performed with a uniform mesh of 576 special points and a Gaussian energy smearing scheme with a width of 0.2 $\mathrm{eV}$. The surface is presented by nine-layer Pd slabs separated by five layers of vacuum. This thickness is quite sufficient to avoid a strong interaction between the two surfaces of a slab: the splitting of the $\bar{\Gamma}$ surface state consists of only $0.25 \mathrm{eV}$. For comparison with experimental data we average the energies of these two states. Atomic positions inside the $\operatorname{Pd}(111)$ planes are fixed at the theoretical lattice parameter, $a$ $=7.34$ a.u., which is close to the experimental value. ${ }^{21}$ The calculated relaxation of the $\operatorname{Pd}(111)$ layers shows a very small contraction of the outermost interlayer spacing (about $0.15 \%$ ) relative to the bulk distance, that is, consistent with the experimental expansion of $0.9 \pm 1.3 \% .^{22}$

The phonon-induced lifetime broadening of an electron state with momentum $\mathbf{k}_{\nu}$ and energy $\epsilon_{\mathbf{k}_{\nu}}$ is related to the Eliashberg spectral function $\alpha^{2} F$ through the integral over all the scattering events that conserve energy and momentum ${ }^{23}$ (we use atomic units, i.e., $e^{2}=\hbar=m_{e}=1$ )

$$
\begin{aligned}
\Gamma_{e-p h}\left(\mathbf{k}_{\nu}\right)= & 2 \pi \int_{0}^{\omega_{\mathrm{m}}} \alpha^{2} F^{\mathrm{E}}\left(\mathbf{k}_{\nu} ; \omega\right)\left[1+n(\omega)-f\left(\epsilon_{\mathbf{k}_{\nu}}-\omega\right)\right] \\
& +\alpha^{2} F^{\mathrm{A}}\left(\mathbf{k}_{\nu} ; \omega\right)\left[n(\omega)+f\left(\epsilon_{\mathbf{k}_{\nu}}+\omega\right)\right] d \omega .
\end{aligned}
$$

Here, $f$ and $n$ are the Fermi and Bose distribution functions, and $\omega_{\mathrm{m}}$ is the maximum phonon frequency. $\alpha^{2} F^{\mathrm{E}(\mathrm{A})}\left(\mathbf{k}_{\nu} ; \omega\right)$ is the electron state-dependent Eliashberg spectral function corresponding to phonon emission (E) and absorption (A) processes. The state-dependent $e$-ph coupling parameter is just the first reciprocal moment of the Eliashberg function

$$
\lambda\left(\mathbf{k}_{\nu}\right)=\int_{0}^{\omega_{\mathrm{m}}} \frac{\alpha^{2} F^{\mathrm{E}}\left(\mathbf{k}_{\nu} ; \omega\right)+\alpha^{2} F^{\mathrm{A}}\left(\mathbf{k}_{\nu} ; \omega\right)}{\omega} d \omega .
$$

The evaluation of the $e-e$ scattering contribution to the linewidth, $\Gamma_{e-e}$, was performed within the first-principles $\mathrm{GW}$ self-energy formalism. Using the calculated ground-state electronic structure we evaluate $\Gamma_{e-e}$ of an electron (hole) in the state $\psi_{\mathbf{k}_{\nu}}(\mathbf{r})$ as

$$
\begin{aligned}
\Gamma_{e-e}\left(\mathbf{k}_{\nu}\right)= & -2 \sum_{\nu^{\prime}, \mathbf{k}, \mathbf{q}}, \sum_{\mathbf{G}, \mathbf{G}^{\prime}} B_{\mathbf{k}_{\nu}, \mathbf{k}_{\nu^{\prime}}}(\mathbf{q}+\mathbf{G}) \\
& \times \operatorname{Im} W_{{\mathbf{G}, \mathbf{G}^{\prime}}^{\prime}}\left(\mathbf{q},\left|\epsilon_{\mathbf{k}_{\nu}}-\epsilon_{\mathbf{k}_{\nu^{\prime}}}\right|\right) B_{\mathbf{k}_{\nu^{\prime}} \mathbf{k}_{\nu^{\prime}}}^{*}\left(\mathbf{q}+\mathbf{G}^{\prime}\right) .
\end{aligned}
$$

Here $\mathbf{G}$ and $\mathbf{G}^{\prime}$ are the reciprocal-lattice vectors, $W_{\mathbf{G}, \mathbf{G}^{\prime}}(\mathbf{q}, \omega)$ is the Fourier transform of the screened interaction $W\left(\mathbf{r}, \mathbf{r}^{\prime}, \omega\right)$, and $B_{\mathbf{k}_{v}, \mathbf{k}_{\nu}}(\mathbf{q}+\mathbf{G})$ are coefficients

$$
B_{\mathbf{k}_{\nu}, \mathbf{k}_{\nu^{\prime}}}(\mathbf{q}+\mathbf{G})=\int \psi_{\mathbf{k}_{\nu}}^{*}(\mathbf{r}) e^{i(\mathbf{q}+\mathbf{G}) \mathbf{r}} \psi_{\mathbf{k}_{\nu^{\prime}}}(\mathbf{r}) d \mathbf{r} .
$$

More details of the GW calculation can be found in Ref. 5 .

\section{CALCULATION RESULTS AND DISCUSSION}

The calculated energy of the unoccupied surface state at $\bar{\Gamma}$ is $1.21 \mathrm{eV}$ above $E_{\mathrm{F}}$ (Fig. 1). Our measured value at $450 \mathrm{~K}$ is $1.31 \mathrm{eV}$ with a temperature-dependent shift of $0.12 \mathrm{meV} / \mathrm{K}$
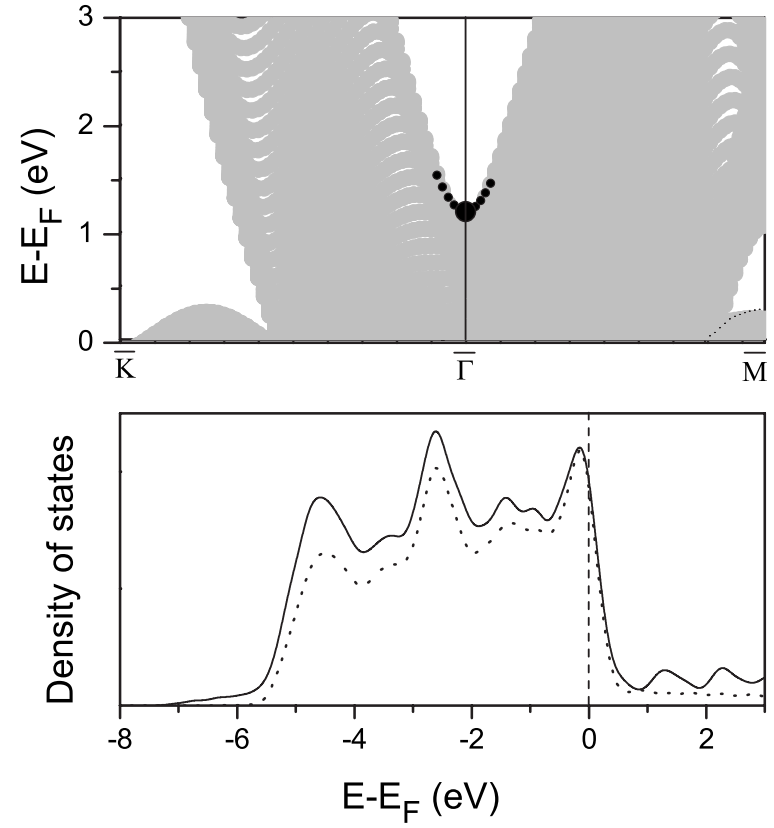

FIG. 1. (Upper panel) The Shockley surface electronic state (black circles) on Pd(111). The gray background represents bulk Pd bands projected onto the two-dimensional Brillouin zone. (Lower panel) The calculated total density of electronic states, DOS (solid line) and DOS of $d$ states only (dotted line) for a nine-layer Pd slab.

(see also Fig. 4). This yields at $T=0$ a value of $1.26 \mathrm{eV}$ in agreement with the calculation and previous results. ${ }^{16}$ First, we calculated exactly the electron-phonon matrix elements using 576 wave vectors $\mathbf{q}$ in the SBZ. Then, to check the convergence the summation was carried out on a denser mesh of $2304 \mathbf{q}$ points. For the additional $\mathbf{q}$ points, the matrix elements were calculated using a Fourier interpolation scheme for the change in the self-consistent potential with respect to atomic displacements as well as for the dynamical matrices.

Figure 2 shows $\alpha^{2} F\left(\mathbf{k}_{\nu} ; \omega\right)$ for the unoccupied $\bar{\Gamma}$ surface state. Since the contributions from phonon emission and adsorption processes nearly coincide, only the average

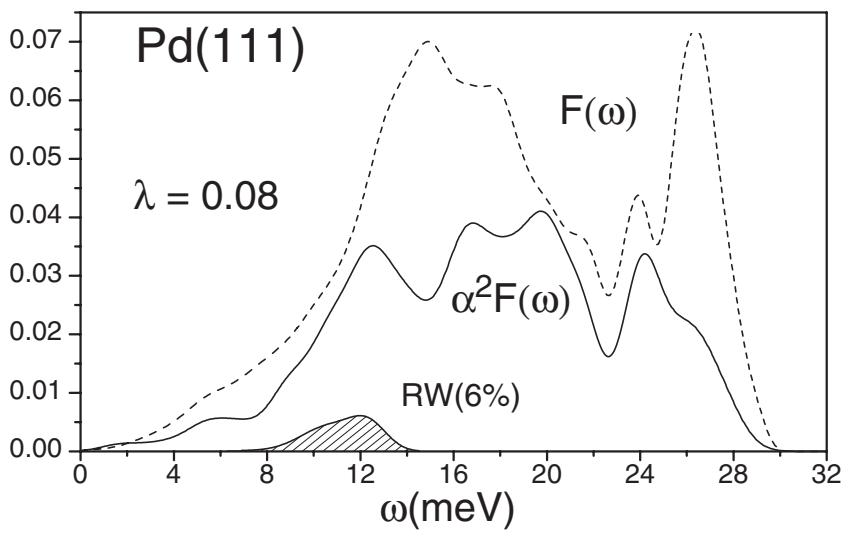

FIG. 2. Electron-phonon Eliashberg spectral function $\alpha^{2} F(\omega)$ for the $\bar{\Gamma}$ unoccupied surface state. The contribution from the Rayleigh surface mode is indicated by hatched area. Phonon density of states for a nine-layer Pd slab, $F(\omega)$, is shown by dashed line. 
TABLE I. Electron-phonon coupling parameters for surface electronic states at $\bar{\Gamma}$ (Refs. 24-27). The surface-state energies $E$ are shown with respect to the Fermi level. Also $\lambda$ averaged over momentum at $E_{\mathrm{F}}$ of bulk Al, Mg, Pd (Ref. 17), and noble metals (Ref. 23) are presented.

\begin{tabular}{lcccccc}
\hline \hline & $\mathrm{Ag}(111)$ & $\mathrm{Cu}(111)$ & $\mathrm{Au}(111)$ & $\mathrm{Al}(001)$ & $\operatorname{Mg}(0001)$ & $\operatorname{Pd}(111)$ \\
\hline$E(\mathrm{eV})$ & -0.04 & -0.44 & -0.5 & -2.8 & -1.7 & 1.26 \\
$\lambda$ & 0.12 & 0.16 & 0.11 & 0.51 & 0.28 & 0.08 \\
$\lambda\left(E_{F}\right)$ & 0.10 & 0.14 & 0.14 & 0.44 & 0.30 & 0.4 \\
\hline \hline
\end{tabular}

$\alpha^{2} F\left(\mathbf{k}_{\nu} ; \omega\right)$ is shown. We calculated $\lambda$ for both split surface states at $\bar{\Gamma}$ and obtained $\lambda_{\bar{\Gamma}}=0.07$ for the lower state and $\lambda_{\bar{\Gamma}}=0.09$ for the upper state. Extrapolating these results to the thick films (semi-infinite crystal) we take the average value of $\lambda_{\bar{\Gamma}}=0.08$. The obtained $\lambda_{\bar{\Gamma}}$ is very small and differs drastically from the $e$-ph coupling parameter averaged over momenta at the Fermi energy of the nine-layer film of $\operatorname{Pd}(111)$ and of bulk Pd, $\lambda_{E_{\mathrm{F}}}=0.4 .^{17}$

As a rule, the strength of the $e$-ph interaction in a Shockley surface state is close to $\lambda$ at the Fermi energy of the corresponding bulk material (see Table I). On the (111) surface of noble metals, the Shockley states lie just below $E_{\mathrm{F}}$ and well inside the surface-projected band gap. As a result, their wave functions are mostly localized near the surface and the contribution to the e-ph coupling coming from surface-phonon modes is important. ${ }^{26}$ The Shockley state at $\operatorname{Pd}(111)$ resembles much the $\bar{\Gamma}$ surface state on $\operatorname{Al}(001){ }^{25}$ Both states lying very close to the bottom of the band gap at $\bar{\Gamma}$ are characterized by a deep penetration into the bulk. In both cases, the $e$-ph coupling is determined by bulk phonons and the contribution coming from the surface Raleigh modes is very small. It does not exceed $6 \%$ for the surface state on $\operatorname{Pd}(111)$ (see Fig. 2). However, like in the case of noblemetal (111) surfaces, $\lambda_{\Gamma}^{-}$for the $\mathrm{Al}(001)$ surface state is close to that at $E_{\mathrm{F}}$ of bulk Al though the state is much deeper in energy ${ }^{25}$ The strength of the $e$-ph coupling for the Shockley state on $\mathrm{Pd}(111)$ is closer to that of image-potential states, especially, when the latter lies close to the band edge, e.g., on the $\mathrm{Cu}(111)$ surface. ${ }^{28}$ Such negligible values of $\lambda$ for the image states were attributed to a small penetration of the states into the bulk, e.g., for $\operatorname{Pd}(111)$ the penetration of the first image-potential state is $4.6 \%{ }^{16}$ However, the situation with the unoccupied surface state on $\operatorname{Pd}(111)$ is quite different. It is located almost completely (90\%) inside the bulk. A plausible reason could be an abrupt decrease in the density of states above $E_{\mathrm{F}}$ and, correspondingly, the number of electronic states which may participate in the $e$-ph coupling (see Fig. 1). Though such a relation is not evident. The values of DOS at the energies of the $\operatorname{Pd}(111)$ surface state and the $\bar{\Gamma}$ surface electronic states on $\mathrm{Al}(001)$ and $\mathrm{Mg}(0001)$ do not differ substantially $(\sim 2,2.4$, and 3.4 states/Ry/atom/spin, respectively) unlike the values of $\lambda: 0.08,0.51$, and 0.30 , respectively. ${ }^{24,25}$ On the other hand, the calculations for the $\mathrm{Al}(001)$ surface showed that there was no direct relationship between the value of DOS and $\lambda$, e.g., the density of states at the energy of the surface state at the $\bar{X}$ point is half as large than that at the $\bar{\Gamma}$ point while $\lambda_{\bar{X}}=0.78$.
The evaluation of the $e-e$ scattering contribution to the linewidth gives $\Gamma_{e-e}=29 \mathrm{meV}$ for the surface state. That is smaller than $37 \mathrm{meV}$ obtained from the one-dimensional calculation. ${ }^{16}$ This difference can be attributed to use of $a b$ initio electronic structure that includes $d$ states just above $E_{\mathrm{F}}$ in the electron transitions to final states and as well as in the screening.

Figure 3 shows the calculated linewidth of the unoccupied surface state as a function of temperature by a solid line. The temperature-independent linewidth, $\Gamma_{e-e}=29 \mathrm{meV}$, has been added and is indicated by the dashed line. We also show in Fig. 3 two data sets for the intrinsic linewidth derived from two-photon photoemission. The data were obtained by exciting the unoccupied surface state by a photon with energy $h \nu=1.55 \mathrm{eV}$ from occupied states below the Fermi energy $E_{\mathrm{F}}$ (see inset of Fig. 4and Ref. 16). A second photon with energy $3 h \nu=4.65 \mathrm{eV}$ lifts the energy above the vacuum level $E_{v a c}$. Electrons emitted along the surface normal are detected by a hemispherical energy analyzer. ${ }^{16}$ The kineticenergy scale in Fig. 4 refers to the analyzer and the work function can be obtained by adding the analyzer work function of $4.32 \mathrm{eV}$ to the low-energy cutoff of the spectra. The decrease in $-0.11 \mathrm{meV} / \mathrm{K}$ is similar to values for other surfaces..$^{29}$ The spectra in Fig. 4 show an intensity decrease and linewidth increase with temperature for the peak assigned to the Shockley surface state. ${ }^{16}$ Spectra were fitted using a Lorentzian for the intrinsic linewidth convoluted with a Gaussian accounting for the experimental resolution

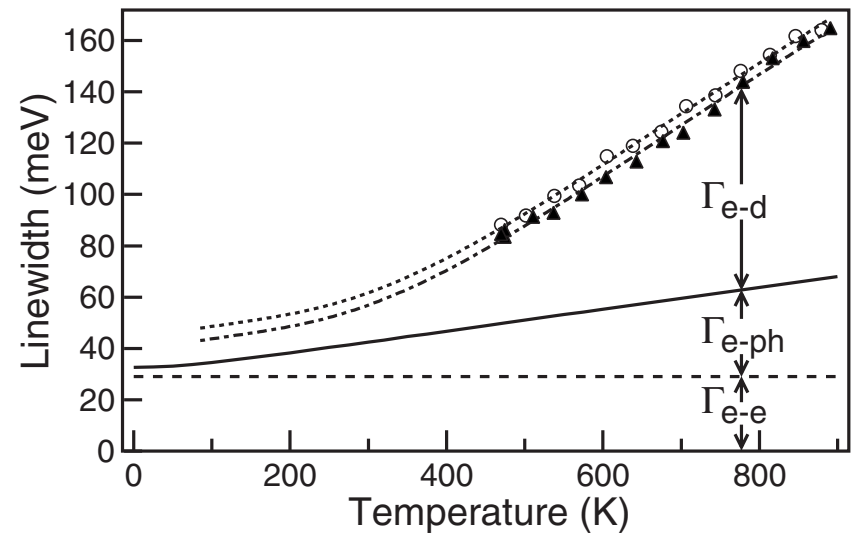

FIG. 3. Temperature dependence of the lifetime broadening for the unoccupied surface state at $\bar{\Gamma}$. The solid curve shows the calculated electron-phonon contribution plus the electron-electron contribution (dashed line). The symbols present two experimental data sets with the corresponding fits including the contribution by thermally generated defects. 


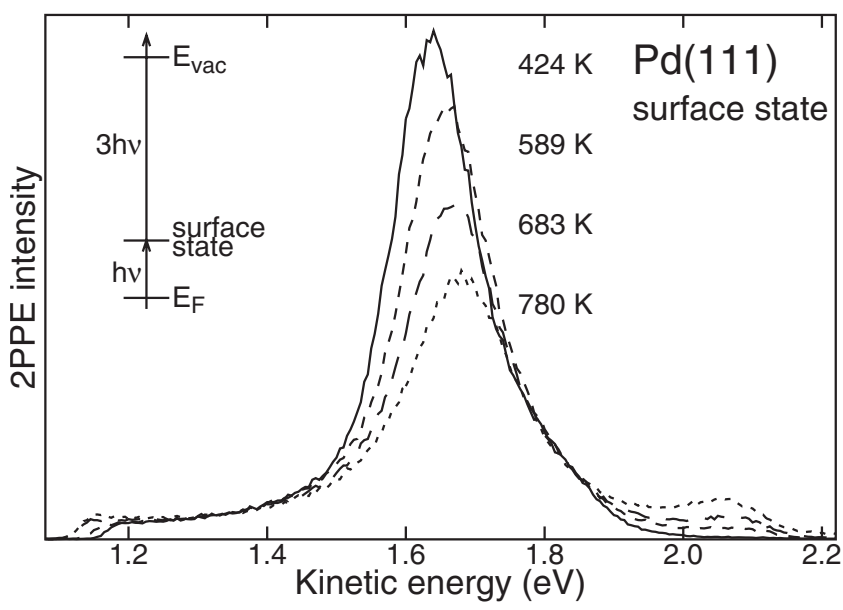

FIG. 4. Two-photon photoemission spectra for the unoccupied surface state at the $\bar{\Gamma}$ symmetry point for different temperatures. The kinetic energy is relative to the vacuum level of the analyzer. The inset shows the energy diagram of the two-photon photoemission process.

of the analyzer and the bandwidth of the laser pulses. The experimental data in Fig. 3 can be fitted using the hightemperature approximation $\Gamma_{e-p h}=2 \pi \lambda k T$ (Refs. 23 and 30) with $\lambda=0.38 \pm 0.05$. This value is much larger than the calculated one and would extrapolate the linewidth to negative values for $T=0$. These variances can be resolved by including the electron scattering due to thermally activated defects following Ref. 30. This contribution is modeled by an added term $\Gamma_{e-d}=C \exp \left(-E_{a} / k T\right)$ to the expression for the temperature-dependent linewidth. The activation energy $E_{a}$ and $\lambda$ cannot be fitted simultaneously in the available temperature range, so we used the calculated value $\lambda=0.08$. The resulting fits are shown as dotted and dash-dotted lines in Fig. 3. The obtained activation energy, $115 \pm 13 \mathrm{meV}$ is in the range of the values for $\mathrm{Au}(111)(81 \mathrm{meV})$ and $\mathrm{Al}(100)$ $(170 \mathrm{meV}){ }^{30}$ The value of the prefactor $C=390 \pm 20 \mathrm{meV}$ compares similarly. When the exponential factor is interpreted as defect concentration the obtained value corresponds to $4 \mathrm{meV}$ linewidth increase for $1 \%$ defects. This value is similar to the linewidth increase due to adatoms for image-potential states on $\mathrm{Cu}(100){ }^{31}$

Measurements of the linewidth for the image-potential state on $\mathrm{Pd}(111)$ as a function of temperature using a straight-line fit give also a rather high value for $\lambda=0.14$. For the image-potential state on $\mathrm{Cu}(111), \lambda=0.06$ has been measured which is attributed to a rather strong coupling to bulk bands at an energy near the band edge ${ }^{28}$ due to the significant overlap of the image-potential state and bulk states wave functions. For $\mathrm{Ag}(100)$ and $\mathrm{Cu}(100)$ which have band gaps similar to $\operatorname{Pd}(111) \lambda=0.01$ has been calculated ${ }^{32}$ and no temperature dependence of the linewidth has been observed, ${ }^{33}$ respectively. A consistent fit of the data for the imagepotential state on $\operatorname{Pd}(111)$ assuming $\lambda=0$ can be obtained using the thermally induced defect model with an activation energy $E_{a}=74 \pm 15 \mathrm{meV}$ and a prefactor $C=135 \pm 12 \mathrm{meV}$. Because the image-potential state is measured with the available photon energy close to the Fermi edge the linewidths might be underestimated somewhat. This would lead in turn to a larger value for $E_{a}$ and explains the deviation to the value obtained for the Shockley surface state.

The linewidth extrapolated to $T=0$ for the best sample preparations is $39 \pm 5 \mathrm{meV}$ which has to be compared to the calculated contribution from electron-electron scattering, $\Gamma_{e-e}=29 \mathrm{meV}$. Adding the contribution from the electronphonon scattering, $\Gamma_{e-p h}=4 \mathrm{meV}$ at $T=0$, to the calculated $\Gamma_{e-e}$ yields a reasonable agreement between the experiment and calculation. For the image-potential state a similar agreement is reached with a calculated value of $27 \mathrm{meV}$ (Ref. 16) compared to the experimental value of $24 \pm 6 \mathrm{meV}$ for the linewidth extrapolated to $T=0$. Time-resolved measurements at $450 \mathrm{~K}$ (Ref. 16) gave for the surface state $\hbar / \tau$ $=54 \pm 13 \mathrm{meV}$ which includes besides $\Gamma_{e-e}$ also contributions from phonon- and possible defect-induced interband scattering from the surface state to bulk bands. The measured value is in good agreement with the calculated $\Gamma_{e-e}+\Gamma_{e-p h}$ $=29+19 \mathrm{meV}$ result. The measured lifetime broadening $\hbar / \tau$ for the surface state increases with increasing temperature. This observation as well as the comparison of the different linewidth contributions at $450 \mathrm{~K}$ indicate that phonon- and defect-induced scattering to bulk bands is about a factor of 5 smaller than the corresponding elastic intraband scattering.

\section{SUMMARY}

We have presented the results of an $a b$ initio study and time-resolved two-photon photoemission measurements of the electron linewidths for the Shockley surface state on $\operatorname{Pd}(111)$. The calculations show that the $e$-ph interaction is mostly determined by coupling of bulk electronic states to bulk-phonon modes. This interaction results in an unusually small $e$-ph coupling parameter $\lambda$ at the $\bar{\Gamma}$ surface state due to a low density of bulk electron states at the surface-state energy. Time-resolved two-photon photoemission measurements taking into account thermally created defects confirm the calculated results.

\section{ACKNOWLEDGMENTS}

This work was partly supported by the University of the Basque Country (Grant No. GIC07IT36607), the Departamento de Educación del Gobierno Vasco, and the Spanish Ministerio de Ciencia y Tecnología (MCyT) (Grant No. FIS200766711C0101). The work of V.M.S. is sponsored by the IKERBASQUE FOUNDATION. 
${ }^{1}$ L. Bartels, F. Wang, D. Möller, E. Knoesel, and T. F. Heinz, Science 305, 648 (2004).

${ }^{2}$ Laser Spectroscopy and Photochemistry on Metal Surfaces, edited by H. L. Dai and W. Ho (World Scientific, Singapore, 1995).

${ }^{3}$ H. Nienhaus, Surf. Sci. Rep. 45, 1 (2002).

${ }^{4}$ I. Zutić, J. Fabian, and S. Das Sarma, Rev. Mod. Phys. 76, 323 (2004).

${ }^{5}$ E. V. Chulkov, A. G. Borisov, J.-P. Gauyacq, D. Sànchez-Portal, V. M. Silkin, V. P. Zhukov, and P. M. Echenique, Chem. Rev. (Washington, D.C.) 106, 4160 (2006).

${ }^{6}$ Th. Fauster, M. Weinelt, and U. Höfer, Prog. Surf. Sci. 82, 224 (2007).

${ }^{7}$ B. A. McDougall, T. Balasubramanian, and E. Jensen, Phys. Rev. B 51, 13891 (1995).

${ }^{8}$ F. Theilmann, R. Matzdorf, G. Meister, and A. Goldmann, Phys. Rev. B 56, 3632 (1997).

${ }^{9}$ R. Matzdorf, Surf. Sci. Rep. 30, 153 (1998).

${ }^{10}$ A. Nojima, K. Yamashita, and B. Hellsing, Appl. Surf. Sci. 254, 7938 (2008); J. Phys.: Condens. Matter 20, 224017 (2008)

${ }^{11}$ T. Valla, A. V. Fedorov, P. D. Johnson, and S. L. Hulbert, Phys. Rev. Lett. 83, 2085 (1999).

${ }^{12}$ J. Kröger, Appl. Phys. A: Mater. Sci. Process. 87, 345 (2007).

${ }^{13}$ T. K. Kim, T. S. Sørensen, E. Wolfring, H. Li, E. V. Chulkov, and Ph. Hofmann, Phys. Rev. B 72, 075422 (2005).

${ }^{14}$ P. Hofmann, Prog. Surf. Sci. 81, 191 (2006).

${ }^{15}$ E. W. Plummer, J. Shi, S.-J. Tang, E. Rotenberg, and S. D. Kevan, Prog. Surf. Sci. 74, 251 (2003).

${ }^{16}$ A. Schäfer, I. L. Shumay, M. Wiets, M. Weinelt, Th. Fauster, E. V. Chulkov, V. M. Silkin, and P. M. Echenique, Phys. Rev. B 61, 13159 (2000).

${ }^{17}$ S. Y. Savrasov and D. Y. Savrasov, Phys. Rev. B 54, 16487 (1996); I. Yu. Sklyadneva, A. Leonardo, P. M. Echenique, S. V. Eremeev, and E. V. Chulkov, J. Phys.: Condens. Matter 18, 7923 (2006)

${ }^{18}$ R. Heid and K.-P. Bohnen, Phys. Rev. B 60, R3709 (1999).

${ }^{19}$ B. Meyer, C. Elsässer, and M. Fähnle, FORTRAN90 program for mixed-basis calculations for crystals, Max-Planck-Institut für Metallforschung, Stuttgart.
${ }^{20}$ D. Vanderbilt, Phys. Rev. B 32, 8412 (1985).

${ }^{21}$ C. Kittel, Introduction to Solid State Physics (Wiley, New York, 1961)

${ }^{22}$ H. Ohtani, M. A. van Hove, and G. A. Somorjai, Surf. Sci. 187, 372 (1987).

${ }^{23}$ G. Grimvall, The Electron-Phonon Interaction in Metals (NorthHolland, Amsterdam, New York, Oxford, 1981).

${ }^{24}$ A. Leonardo, I. Yu. Sklyadneva, V. M. Silkin, P. M. Echenique, and E. V. Chulkov, Phys. Rev. B 76, 035404 (2007).

${ }^{25}$ I. Yu. Sklyadneva, E. V. Chulkov, and P. M. Echenique, J. Phys.: Condens. Matter 20, 165203 (2008).

${ }^{26}$ A. Eiguren, B. Hellsing, E. V. Chulkov, and P. M. Echenique, Phys. Rev. B 67, 235423 (2003).

${ }^{27} \mathrm{We}$ comment here about the model calculation result, $\lambda=0.23$, for the $\bar{\Gamma}$ surface state on $\mathrm{Al}(001)$ (Ref. 26) and the $a b$ initio calculation value, $\lambda=0.51$ (Ref. 25 ). While at $E_{\mathrm{F}}$ both computations give fairly close $\lambda$ values [0.55 (Ref. 26) and 0.44 (Ref. $25)]$ at the $\bar{\Gamma}$ surface state the model calculation $\lambda$ is significantly smaller than the $a b$ initio one. We attribute this discrepancy to the use of the Ashcroft pseudopotential in the model calculation. The one-parameter Ashcroft pseudopotential reproduces correctly the electron scattering at the Fermi surface while it is essentially less accurate for energies far from $E_{\mathrm{F}}$. It is the case of the $\bar{\Gamma}$ surface state on $\mathrm{Al}(001)$.

${ }^{28}$ E. Knoesel, A. Hotzel, and M. Wolf, J. Electron Spectrosc. Relat. Phenom. 88-91, 577 (1998).

${ }^{29}$ T. Durakiewicz, A. J. Arko, J. J. Joyce, D. P. Moore, and S. Halas, Surf. Sci. 478, 72 (2001).

${ }^{30}$ M. F. Jensen, T. K. Kim, S. Bengió, I. Yu. Sklyadneva, A. Leonardo, S. V. Eremeev, E. V. Chulkov, and Ph. Hofmann, Phys. Rev. B 75, 153404 (2007).

${ }^{31}$ K. Boger, T. Fauster, and M. Weinelt, New. J. Phys. 7, 110 (2005); M. Hirschmann and Th. Fauster, Appl. Phys. A: Mater. Sci. Process. 88, 547 (2007).

${ }^{32}$ A. Eiguren, B. Hellsing, E. V. Chulkov, and P. M. Echenique, J. Electron Spectrosc. Relat. Phenom. 129, 111 (2003).

${ }^{33}$ M. Weinelt, J. Phys.: Condens. Matter 14, R1099 (2002). 